\title{
Em Direcção ao Museu Ubíquo: uma realidade ou ainda uma utopia?
}

Maria José Messias 1

\section{Resumo:}

No presente artigo, elaborado no âmbito da investigação de doutoramento que me encontro a realizar, pretende-se analisar o impacto das novas tecnologias de comunicação e informação na mudança do paradigma comunicacional das instituições museológicas, no sentido de priorizarem uma museologia mais presente, participativa, e relevante.

\section{Palavras chave:}

Museologia, media sociais, participação, acervos digitais, comunicação.

\section{Abstract}

In the present article, written as part of the doctoral research that I am conducting is intended to analyze the impact of new technologies of communication and information in the change of the communication paradigm of museum institutions, to prioritize one ubiquitous, participatory, and relevant museology. Keywords:

Museology, social media participation, digital collections, communication

1 Mestre e Doutoranda em Museologia na Universidade Lusófona de Humanidades e Tecnologias, mariamessias@hotmail.com 
"As ferramentas de comunicação não se tornam socialmente interessantes até se tornarem tecnologicamente aborrecidas (...) É quando uma tecnologia se torna normal, depois ubíqua e finalmente tão generalizada que se torna invisível, que as mudanças profundas ocorrem." (Shirky 2008:105).

Um adjectivo recorrentemente na literatura relativa às Tecnologias de Informação e Comunicação (TIC), é a sua ubiquidade na sociedade contemporânea ocidental. Ser ubíquo, é estar ao mesmo tempo em toda a parte, difundido em todo o lado, geral, universal e omnipresente. A capacidade de penetração das TIC em todas as esferas da vida humana, tornouas ubíquas, esta é uma das suas características mais marcantes, que revolucionou o modo de vida actual, alterando a forma como geramos e acedemos à informação e à cultura e como interagimos uns com os outros. A literacia digital assume um papel cada vez mais importante na vida das pessoas à medida que os media e as tecnologias continuam cada vez mais a afectar a forma como as pessoas trabalham, socializam, comunicam, despendem o seu tempo de lazer, aprendem e partilham o conhecimento (Macdonald \& Alsford, 1997). As tecnologias da informação e da comunicação, são muito mais do que ferramentas, elas centralizam, suportam e moldam as nossas formas de pensar e de criar, a cultura é habitada e elaborada pela tecnologia.

Vivemos hoje num mundo globalizado, tanto a nível económico, como tecnológico e cultural, assente na transacção de bens e serviços numa rede complexa de dependências mútuas, de grupos financeiros e tratados internacionais de natureza política, 
comercial e diplomática - o mundo sem fronteiras dos capitais, das multinacionais e do ciberespaço - moldado pelas lógicas, do individualismo e do consumismo (Lipovetsky \& Serroy, 2008), onde as redes e os fluxos culturais circulam e interagem a uma escala planetária a par do rápido desenvolvimento e expansão das tecnologias de comunicação e informação.

A sociedade contemporânea, cada vez mais globalizada, diversa e multicultural, vai caracterizar-se por uma ausência de referentes de identidade, de estabilidade e de continuidade, fruto das migrações, da mobilidade geográfica e social e da profusão de informação difundida pelos media à escala mundial, dando origem à sobreposição de diferentes culturas e ao surgimento de culturas híbridas, e às consequentes tensões e questionamentos daí resultantes perante a ausência de referentes comuns quanto a noções estruturantes de identidade, cultura, classe, raça, nacionalidade ou sexo. As instituições culturais, e em particular os museus, por trabalharem com estes conceitos, cada vez mais móveis e problemáticos, de identidade, de memória e de valores, actuam num campo particularmente sensível e delicado, em que se torna necessário atender a múltiplas demandas, conciliar e dar expressão a diferentes vozes e interesses de audiências cada vez mais diversificadas e exigentes. Para tal é necessário o museu estar aberto, atento e permeável às preocupações, desejos, expectativas e iniciativas dos seus públicos, nesse sentido as TIC, pela sua capacidade de facilitarem o diálogo entre a instituição e o seus utilizadores, possibilitando novas formas de participação e de co-construção de acervos patrimoniais e o seu debate, podem prestar um contributo indispensável.

Ao longo da história, os museus têm sabido adaptar-se a diferentes dinâmicas e fenómenos, reflectindo o contexto sociocultural em que estão inseridos, e a inclusão e apropriação 
das tecnologias de informação e comunicação, e das novas possibilidades que estas oferecem para a prática museológica não foi excepção. A computorização, iniciado em larga escala com a disseminação do computador pessoal nos anos 80, a digitalização, o acesso remoto à informação disponibilizada pela Web, e o surgimento de novos canais de comunicação deram origem a uma mudança estrutural na forma como os museus vão interpretar as suas colecções, na forma como a informação é tratada dentro das instituições, na pesquisa, conservação e documentação, e de como vai ser comunicada às audiências, dentro do espaço do museu e online, e também a forma como as audiências interagem com os museus.

A disseminação e crescimento exponencial de tecnologias móveis e ubíquas, tornou possível aceder, não só dentro do espaço do museu, mas remotamente, a qualquer um, a qualquer hora e em qualquer lugar, aos seus conteúdos. 0 museu ficou presente, e acedível por todos, ao mesmo tempo e em toda a parte, difundido em todo o lado, e omnipresente na vida das pessoas. Será que caminhamos também no sentido de um museu ubíquo, que adopta as características e se transfigura em consequência dos media tecnológicos que emprega e que se tornaram transversais a todas as áreas da prática museológica? Um museu presente, constante e relevante na vida das pessoas? Que estratégias e acções tem vindo a ser desenvolvidas pelos museus no sentido de fortalecer a sua relação com as audiências, estimulando a participação e o envolvimento das comunidades? Como têm sabido lidar as instituições com a ascensão do poder da informação e com a partilha de poder necessária a estabelecer curadorias colaborativas? São algumas das questões a explorar. 
Para Foucault (1998:182) os museus apresentam-se como locais de acumulação perpétua e indefinida do tempo e do espaço, constituem-se como arquivos gerais que ambicionam conter todos os tempos, todas as épocas, todas e formas e gostos num só lugar, um local de todos os tempos e ele próprio fora do tempo e abrigado da sua erosão. À semelhança da ideia de museu de Foucault, o museu ubíquo configura-se como um espaço paralelo, um arquivo de acumulação patrimonial, do tangível e intangível, em constante actualização, e com capacidade de operar em todos os tempos e lugares, flexível, aberto à participação, partilha e criação colaborativa, procurando novos canais para a mediação e o diálogo. Nos anos recentes temos assistido a uma concretização ainda tímida, mas gradual desta ideia, à medida que a tecnologia evoluí e se dissemina dentro das instituições culturais, na sociedade e na vida das pessoas, esbatendo os domínios do espaço físico e do virtual, do público e do privado, dando origem a novos espaços híbridos de partilha, confronto, diálogo e discussão. Segundo Sumption (2006) o museu ubíquo vai tirar partido das inúmeras tecnologias e técnicas que possam alimentar a relação simbiótica entre os domínios físicos e virtuais e não estabelece diferença entre visitantes físicos ou virtuais, vai procurar na tecnologia todas as oportunidades que suportem o uso continuo e cíclico do completo manancial de informação do museu. Esta aproximação holística significa que os visitantes online são encorajados a visitar o espaço físico da instituição e os visitantes desta são encorajados a explorar a informação online. Ambos os domínios se completam e interagem no todo da experiência, dando origem àquilo que Barry (2006) denomina de circulo virtuoso. 
Nas últimas décadas o campo de actuação cultural, alterou-se e ampliou-se, dando origem a novos métodos de fazer e promover a cultura, a participação e a cidadania. A noção de património expandiu-se, valorizando de igual modo o património tangível (o material) e o património intangível (o imaterial) que vai englobar o emergente património digital, o "objecto digital" inicialmente encarado com suspeição por poder entrar em competição ou pôr em causa a supremacia do "objecto museológico" real, original e autentico dentro da instituição. Verifica-se também uma maior abertura à cultura popular e à história de diferentes classes sociais e etnias, antes negligenciadas pelos museus. 0 ênfase transitou da colecção para as pessoas - os visitantes/utilizadores - isto é, os museus passam de ser acerca de algo para serem para alguém (Weil, 1999), sublinhando o facto de estes providenciarem um serviço público, pelo que é necessário priorizarem as necessidades das audiências, a sua representação dentro da instituição e a qualidade da experiência proporcionada. Deste modo alterou-se também o paradigma do museu legislador (autoritário e detentor da verdade ou do saber indiscutível) para o museu intérprete de significados culturais (Ross, 2004), que contribui para a análise e conhecimento da sociedade, excluindo práticas elitista e monovocais, valorizando a transparência na tomada de decisões e estimulando a inclusão e a participação, reconhecendo e incorporando o saber cultural dos visitantes e das comunidades. Procurando-se abolir a divisão entre o museu como espaço ritual e como espaço público, aberto a interpretações multifacetadas constantemente produzidas e reformuladas num processo contínuo e variável de interacção entre o museu, os seus utilizadores e os media. 
Verificou-se também uma importante mudança da ideia de museu como um repositório de objectos para a ideia do museu como um repositório de conhecimento (Marty, 2008), onde a necessidade de providenciar acesso à informação acerca das colecções, dos objectos ou tópicos expostos é imperativa, e a acessibilidade à informação sobre as colecções é tão importante como as próprias colecções (Pearce, 1986). Tal implica que a gestão da informação do museu, as suas práticas e sistemas devam ser reorientados para o exterior, de modo a irem de encontro às necessidades das audiências externas, e não só cumprirem necessidades internas de processamento da informação (Peacock, 2008). Se até à modernidade o acesso a colecções (de arte ou científicas) e à informação era restrito às classes privilegiadas, cuja riqueza ou estatuto o permitia, hoje a disseminação e a facilidade de acesso à informação (às colecções e objectos digitais), e a ubiquidade das tecnologias digitais que o permitem, atinge níveis sem precedentes, possibilitando que recursos antes inacessíveis ao público em geral estejam cada vez mais disponíveis a todos os segmentos da sociedade (Zorich, 2008). A informação avoluma-se, flui e dissemina-se, verificandose também uma maior transparência em relação à forma de como essa informação é produzida (Jones, 2008) e o número de museus multiplica-se.

Huyssen (2014), vai explicar este fenómeno como consequência do medo do esquecimento desencadeado pelo acumular do excesso de memória de uma cultura saturada pelos media, enquanto veículos de todas as formas de memória pessoal, geracional ou pública, o que para o autor gera uma necessidade de trazer todos os passados para o presente, e leva a que o mundo se encontre naquilo que considera de um processo acelerado de musealização. A salvaguarda da memória a 
musealização surgem como baluartes contra a obsolescência e o desaparecimento, vindo contrariar a profunda ansiedade causada pela actual velocidade da mudança e da contracção dos horizontes de tempo e espaço. Contudo uma das grandes ironias da era da informação, o período no qual se tem gerado mais informação e que mais de $90 \%$ dessa informação é produzida em suporte digital (Peacock, 2008), é exactamente a volatilidade deste suporte, e a rapidez com que a tecnologia onde se armazena e gere a parte mais significativa da memória do nosso tempo, e dos tempos passados, fica obsoleta.

Apesar de hoje ser possível ao museu ir além das fronteiras do seu espaço físico, e a acessibilidade (digital) às colecções e a sua audiência potencial e capacidade de alcance, serem agora, virtualmente infinitas, corre-se contudo o risco de simplesmente só se providenciar mais informação para mais pessoas se não se souber tirar partido das capacidades interactivas das TIC, para fomentar o diálogo e a troca de conhecimento, possibilitando aos utilizadores contribuírem para a "construção de significados" (Bradburne 2008), aumentando assim a amplitude de perspectivas da instituição e a sua integração na comunidade e na vida das pessoas. Esta ideia não é nova, nem depende exclusivamente das tecnologias digitais, contudo a crescente inclusão e participação das audiências nas instituições de memória encontra-se intimamente relacionada com as novas possibilidades tecnológicas. 0 desenvolvimento e partilha de conteúdos gerados pelo utilizador, evoluiu à medida que as novas capacidades da web o possibilitaram (Russo, 2012). Não se pode contudo esquecer a responsabilidade social da instituição museu como prestadora de um serviço público na democratização do acesso aos bens culturais, e como tal é também necessário contemplar estratégias comunicacionais 
alternativas para quem não tem ainda acesso ou não domina as tecnologias digitais.

Nas últimas décadas e à medida que as tecnologias de informação evoluíram, e evoluem, aumenta o volume de informação patrimonial, tangível e intangível da Humanidade e da Natureza transposta para o domínio digital, assim como surge novo património que só vai encontrar expressão neste domínio, de que são exemplo as novas formas de arte digital ou media arte, constituindo um vasto e imparável acervo de património digital que não cessa de se ampliar. Assiste-se também à configuração de novas relações de produção e de difusão da arte resultantes da utilização da internet e das novas tecnologias por parte das instituições culturais, e à explosão de novas formas de criatividade produto da intersecção e convergência de várias tecnologias de media, e da interacção reciproca entre industrias e consumidores. As TIC não são apenas ferramentas de comunicabilidade, mas também suporte e meio do objecto artístico, a media arte vai caracterizar-se por ser participativa, dinâmica, não linear, colaborativa, flexível e interactiva, muitos trabalhos permitem a liberdade de navegação, a contribuição e a imersão na obra pelo seu utilizador tornar-se este seu co-autor, pois a sua presença ou interacção, virtual ou real, pode moldá-la e influenciá-la. Christiane Paul (2005) vai diferenciar dentro das categorias de arte digital as que utilizam as tecnologias como ferramenta (para armazenar ou distribuir) e as que as que a utilizam como medium, o que implica que o trabalho artístico é produzido, armazenado e apresentado em formato digital e tira partido das possibilidades inerentes da tecnologia na criação, isso acontece com a produção de instalações interactivas, internet art, projectos de realidade virtual, arte para dispositivos móveis entre outras formas. A media arte vai ampliar e prolongar 
o campo de actuação do artista, para além do espaço da galeria, ou do museu, chegando digitalmente potencialmente a qualquer lugar, o que para Paul exige um museu ubíquo, aberto à criação e à construção de subjectividades.

Se inicialmente o interesse dos museus pela tecnologia assentava sobretudo nas suas possibilidades de gestão documental rapidamente o interesse transitou para as suas capacidades de recriação do acervo, de locais ou objectos patrimoniais, através da modelação computorizada tridimensional ou em Realidade Virtual, que permite a manipulação e a interacção pelos seus utilizadores, sendo a Web rapidamente adoptada pelos museus, pela excelente oportunidade que oferece como canal de veiculação de informação de longo alcance sobre a instituição. 0 website da instituição, afastou-se progressivamente do seu modelo inicial de brochura electrónica, de comunicação unidireccional, focado prioritariamente na divulgação e promoção da instituição em rede de modo a cativar o público online para a visita ao espaço físico da instituição e rapidamente se começou a diversificar a informação oferecida e as plataformas de actuação estenderamse aos blogs, fóruns e redes sociais, disponibilizando serviços online independentes ou complementares à experiência da visita ao espaço físico, mas não necessariamente condicionados por esta e a participação activa dos utilizadores na produção de conteúdos e a interactividade (criar, manipular, partilhar, reinterpretar e distribuir informação em diferentes suportes) foram incentivados com a adopção da Web 2.0., mudando o comportamento dos museus de locais de disseminação de informação para agentes de diálogo.

0 termo Web 2.0, designação atribuída a Tim O’Reilly (Andersen, 2007) pretende identificar a evolução da Web para 
uma plataforma colaborativa, uma nova forma de comunicar e de fazer uso da internet, refere-se sobretudo a um conceito e não a um resultado de uma actualização tecnológica como o nome pode sugerir, e está relacionado com a utilização de aplicativos inteligentes, a proliferação das redes sociais e a possibilidade de utilizar um conjunto de serviços em permanente evolução capazes de promover a participação e a partilha de conteúdos de natureza variada gerados pelos utilizadores ${ }^{2}$.

A Web caracteriza-se por ser um espaço público virtual, democrático e dialógico de interconectividade e aprendizagem social, uma "infra-estrutura de participação" (O’Reily, 2005), na qual não fazem sentido as hierarquias de práticas e estruturas culturais e sociais do museu modernista, impondo um novo modelo de mediação e de construção do conhecimento ao museu, elaborado em conjunto pela instituição e pelos seus utilizadores (Hazan, 2010), trazendo a voz anteriormente passiva da audiência, para o palco da prática museal. Indo encorajar e dar protagonismo às comunidades na interpretação e determinação do significado das colecções dos museus, bem como favorecer a diversificação dos métodos interpretativos, distribuindo e descentralizando a autoridade, ao possibilitar a construção de uma autoridade cooperativa, em que a interpretação se torna interdependente da fusão de múltiplas vozes sobre os objectos,

\footnotetext{
2 Mais recentemente contemplam-se já os termos Web 3.0 e Web 4.0 que vem descrever o desempenho futuro da internet, que se tornará progressivamente mais inteligente e capaz de satisfazer e completar os pedidos dos seus utilizadores, adicionando conteúdos e metadata às aplicações e organizando e processando essa informação de modo a facilitar a sua utilização. Considera-se que a internet tende a evoluir para uma forma mais híbrida, acessível e útil através de recomendações e possibilidades de personalização providenciada pelas tecnologias móveis, e que a Web semântica sairá do campo virtual e se estenderá progressivamente ao mundo físico (internet das coisas).
} 
as colecções e aquilo que representam no espaço do museu (Shahani et al., 2008). Isso significa que a instituição já não vai garantir a consistência da experiencia, mas sim oferecer oportunidades para os seus visitantes co-produzirem experiências. (Simon, 2010).

A actual possibilidade, se bem que ainda escassa, de um individuo criar e expor conteúdos num ambiente cultural tradicionalmente autoritário - como um museu - reflecte o crescente interesse global das instituições e das audiências, pela partilha de experiências individuais e colectivas (Russo et al., 2006). Agora que os museus possuem tecnologia que lhes permite ampliar a intervenção dos visitantes na instituição debatem-se essencialmente com a questão se isso é uma coisa que queiram realmente fazer (Parry, 2008).

No universo digital, o papel do utilizador e do criador da informação estão cada vez mais entrelaçados, tornando difícil distinguir o que separa um do outro. A demarcação entre consumidor/produtor e amador/profissional, esbate-se progressivamente dando origem a actores híbridos no universo dos novos media e da cultura digital, que colapsaram a tradicional corrente de produção, distribuição e consumo (Bannier \& Vleugels, 2010). Alex Bruns (2007) denominou de "produsage" esta forma híbrida de simultaneamente produzir e usar, também definida pelo termo "prosumer" por Toffler (1980), que refere o duplo papel dos utilizadores como produtores e simultaneamente consumidores de conteúdos online. Os visitantes/utilizadores dos museus (seja in loco ou online) já não são passivos receptores de informação, a participação, a mobilidade e a conectividade são expectativas fundamentais da experiência da visita (Rheingold, 2002), o sucesso alcançado por plataformas que cativam milhões de utilizadores como o 
Facebook, Youtube, o Twitter ou o Instagram, que permitem a partilha e inclusão de narrativas e histórias pessoais, o comentar ou a adição de tags, de itens digitais ou o simples expressar do gosto (like) provam-no. As pessoas cada vez mais esperam ser e estar conectadas através das suas experiências, independentemente de onde estão, ou do que estão a fazer, e a tecnologia responde e alimenta esse desejo.

Actualmente o número de visitantes remotos ultrapassa o número de visitantes físicos em grande parte das instituições culturais, os museus servem cada vez mais audiências virtuais globais e diversificadas que não conhecem fisicamente os seus espaços (Zorich, 2008). A relevância da Web é incontornável sobretudo pela sua capacidade de agregar pessoas de diferentes espaços físicos e contextos culturais em torno de redes de interesses comuns. As instituições culturais não podem descurar a sua presença no mundo virtual por onde as pessoas transitam, devendo capitalizar esses recursos e a familiaridade dos indivíduos com as tecnologias, gerindo a sua actuação online, em prol de uma maior proximidade com as audiências, estando atentas àquilo que interessa e preocupa a sociedade e a opinião pública, e receptivas à exposição e à critica que daí pode advir, mas também às propostas fruto da reflexão e desejos das suas audiências, sendo ideal que consigam tirar partido destas para o desenvolvimento de sinergias e projectos futuros.

Com a facilidade actual de aceder ao museu e aos seus conteúdos e de interagir com estes, a partir de qualquer lugar e em qualquer altura, torna-se possível à instituição ficar integrada na vida quotidiana, e o quotidiano das pessoas também se torna parte do museu, esta relação foi viabilizada sobretudo graças à crescente utilização de dispositivos móveis e familiares como os smartphones ou tablets. Muitas instituições culturais e locais 
patrimoniais (centros históricos de cidades, palácios, parques, jardins ou zoos) assim como rotas turísticas disponibilizam online, aplicações (apps), muitas delas gratuitas, que qualquer um pode descarregar e utilizar, e que podem contemplar guias de visita aos locais patrimoniais, com informação detalhada sobre estes, dispositivos de navegação no espaço que facilitam a orientação na galeria ou no exterior, meios de estruturar e personalizar a visita, actividades ou jogos relacionados com os conteúdos patrimoniais que podem incluir tecnologias interactivas de Realidade Aumentada ou Virtual, podendo permitir também carregar ou descarregar informação (downloads ou uploads).

A abertura, a mobilidade, a conectividade, a interacção e a ubiquidade são características cada vez mais presentes na comunicação museal, a actuação do Rijksmuseum ${ }^{3}$ de Amesterdão é disso exemplo, esta instituição vai tirar partido do potencial da Web e das tecnologias móveis para fomentar a relação entre a instituição e o visitante, através dos seus domínios físico e virtual, para tal, disponibiliza guias interactivos de visita às galerias, que permitem explorar a colecção e o edifício em pormenor, recorrendo a animações, a filmes e a diferentes testemunhos sobre a colecção, permitindo também aos utilizadores gravarem informação para referencia futura (bookmarking and retrival), ou seleccionarem as suas obras favoritas, e salvá-las online no Rijksmuseum Studio ${ }^{4}$, uma página Web onde podem, caso o pretendam fazer, efectuar um registo e construir a sua própria colecção online, imprimir, partilhar ou efectuar um download das obras ou criar novos conteúdos a partir destas. A criatividade de cada um é encorajada, através das

\footnotetext{
3 https://www.rijksmuseum.nl

${ }^{4}$ https://www.rijksmuseum.nl/en/rijksstudio
} 
possibilidades da aplicação, existindo inclusive um concurso "Make your own Masterpiece" 5 que premeia a ideia mais criativa, efectuada em qualquer tipo de suporte artístico, inspirada nas obras do museu. Ao ficar registado no Rijksmuseum Studio o visitante/utilizador (in loco ou online) da instituição, vai criar um vinculo com esta que lhe permite dar continuidade à experiência da visita, caso a tenha efectuado, receber informação actualizada e explorar com mais profundidade os recursos da instituição. Um exemplo de procedimento semelhante verifica-se na exposição do Darwin Centre, no Museu de História Natural de Londres, que pretende destacar o papel fulcral da actividade dos investigadores dentro do museu. $\mathrm{Na}$ entrada da exposição é disponibilizado aos visitantes o cartão NaturePlus ${ }^{6}$ que lhes permite guardar conteúdos digitais ao longo do percurso expositivo para posterior exploração online. Mediante registo os utilizadores do cartão acedem a uma página Web personalizada, onde para além de visualizarem os conteúdos que salvaram, podem também aceder a múltipla informação, ver vídeos das expedições e das actividades de investigação realizadas pelo centro, ou participar de fóruns, blogs, ou em inquéritos científicos, ficando conectados a uma comunidade de outros utilizadores e investigadores do museu.

Outros exemplos relevantes são o projecto Antenna Technology Now: Think, Talk, Share ${ }^{7}$ (Pense, Fale e Partilhe) do Museu de Ciência de Londres, em que os visitantes são convidados, in loco através dos quiosques interactivos existentes na exposição ou

\footnotetext{
5 https://news.rijksmuseum.nl/2/4/158/1/fNE0jQnADVhNA6uP_e6CU7 rbtVzdSueAB9ngLJughgjxqqQP7 nbIT C45gzJ2P_Ip

${ }^{6}$ www.nhm.ac.uk/natureplus

7 http://antenna.sciencemuseum.org.uk
} 
online, a participarem e a partilharem o seu ponto de vista, expressando a sua opinião através da votação num inquérito que coloca variadas questões sobre temáticas actuais, tais como: 0 que fazer para parar a propagação do vírus do ébola? 0 que pensam acerca das missões espaciais? Ou da possibilidade de todos poderem possuir um drone com camera? E tanto no espaço expositivo, através de vários ecrãs de grande dimensão onde as estatísticas dos votos dos visitantes podem ser observadas dinamicamente, como online, é possível aceder à constante actualização das respostas e participação dos utilizadores, que se vai constituir como o foco da exposição.

A exposição DNA Amsterdam, no Museu da Cidade de Amesterdão ${ }^{8}$ vai ilustrar o potencial da utilização de aplicativos móveis (apps) para expandir o território de intervenção do museu no exterior, na cidade como território de intervenção museológica, através da oferta online, de diferentes tours multimédia. Estes guias interactivos de visita à cidade vão relacionar os conteúdos expositivos existente na instituição com a cidade real, passada e actual. Os guias incluem mapas, fotos, vídeos, informação áudio e textos, propõem missões e vão colocar questões ligadas à cidade. Pode aceder-se a estas aplicações multimédia através do MuseumApp ${ }^{9}$, no Google Play ou na App Store. A aplicação Canal Stories, vai dar a conhecer as várias histórias dos canais com diferentes sugestões de percursos temáticos para os explorar, é de mencionar que esta aplicação foi desenvolvida em parceria com outras instituições culturais da cidade ${ }^{10}$, já a aplicação Pinkperspectives Tour ${ }^{11}$ vai

\footnotetext{
8 http://www.amsterdammuseum.nl

${ }^{9}$ http://museumapp.nl

10 Casas Cromhout, Casa Geelvinck Hinlopen, Our Lord in The Attic e Museu Willet- Holthusysen
} 
providenciar um percurso focado na herança gay e lésbica da cidade. Estes são dois exemplos da oferta disponível que procura ser abrangente e diversificada não só em relação aos percursos de visita da cidade, como em relação ao tipo de conteúdos apresentados para contar a história e as estórias da cidade, de modo a cativarem públicos com diferentes interesses, e que vão dar continuidade à visita do Museu, ou que podem ser utilizados de forma independente, tirando de qualquer forma partido do trabalho de pesquisa e investigação realizado pelas instituições museológicas da cidade, e gerando laços com elas.

Outros exemplos de expansão da actuação do museu na cidade, graças à utilização de apps para os dispositivos móveis (smartphone ou tablets) dos utilizadores são os guias de visita com percursos temáticos disponibilizados pelo Museu de Londres, o Streetmuseum ${ }^{12}$ ou o Dickens Dark London ${ }^{13}$ que dá a conhecer a Londres sombria da época de Dickens, sob o formato de novelas gráficas interactivas. Ou os guias disponibilizados pelo Memorial do Muro Berlim, ${ }^{14}$ com diferentes temas e percursos ao longo da linha do muro que dividia a cidade e que hoje só permanece em pontos de memória remanescentes da cidade, contando a sua história ou a de eventos a ela associados.

É também de referir a presença constante das instituições citadas, em diferentes redes sociais como o Facebook, Twitter, Pinterest, Spotify ou Flickr, indo destacar como exemplo o canal do Youtube do Museu de Amesterdão ${ }^{15}$ onde é possível aceder a

$11 \mathrm{http}: / /$ waag.org/en/event/museumapp-pinkperspectives

$12 \mathrm{http}: / /$ www.museumoflondon.org.uk/Resources/app/you-are-here-

app/home.html

${ }^{13}$ http://www.museumoflondon.org.uk/Resources/app/Dickens_webpage

/index.html

$14 \mathrm{http}: / /$ berliner-mauer.mobi/touren.html?\&L=1

15 https://www.youtube.com/user/ahmamsterdam 
quase cerca de duas centenas de filmes sobre as colecções, entrevistas, visita a reservas, acções de conservação e restauro, exposições ou eventos da instituição, informação e conteúdos que deste modo ficam facilmente disponíveis aos potenciais interessados.

As infra-estruturas de comunicação e interacção, geradas pelos media sociais, e em particular as redes sociais, actuam como locais de produção cultural e de valores, e podem ser consideradas uma nova geração de práticas patrimoniais vivas (Giaccardi, 2012), vindo a estabelecer novas áreas de investigação. Östman (2010) vai estudar o fenómeno recente da publicação de eventos da vida (life publishing) uma forma de contar a vida diária na Web através de blogs, páginas do Facebook, galerias de fotos ou fóruns, como um novo campo híbrido de práticas autobiográficas. As TIC transformaram as nossas memórias digitais em "artefactos" que podem ser copiados, remisturados e reinterpretados, através dos media aspectos das nossas vidas são capturados e partilhados com os outros que podem anotar e sobrepor informação a esses traços digitais, somando as suas próprias perspectivas às inicialmente publicadas. Este processo possui um cariz essencialmente patrimonial, de preservação da memória do dia-a-dia que nos ajuda a contar histórias acerca de nós e que moldam o nosso sentido de lugar e identidade (Östman, 2010).

Nasce assim assente em práticas curatoriais de partilha, o museu ubíquo do "eu" do "dia-a-dia" , do grupo ou comunidade, na sua página ou local na Web, uma nova forma de curadoria como prática colaborativa e sociotécnica que pode envolver um largo número de pessoas e comunidades, que recorrem às 
tecnologias emergentes para preservar, gerir e partilhar as suas memórias e histórias digitais, colaborando com instituições quando tal é incentivado, mas sobretudo exercendo essas práticas de forma independente e autónoma através das redes sociais. Segundo Liu (2012) a curadoria socialmente distribuída é uma prática sócio-tecnológica que envolve as pessoas, os artefactos culturais, a informação e as tecnologias de comunicação, num um processo curatorial colaborativo de autoria partilhada da memória viva que está a ser preservada, e em que é difícil determinar o seu início e término. É uma abordagem descentralizada, participativa e transparente, que permite a múltiplas pessoas participarem em diferentes momentos do processo curatorial, este é possível quando as memórias e os artefactos são externalizados e partilhados de modo a permitir aos outros o seu uso num processo distribuído através do tempo em que as narrativas de "curadores" passados podem afectar e transformar as futuras narrativas.

Liu vai utilizar como exemplo o caso do acidente de Bhopal na Índia em 1984, o pior acidente industrial, causado por uma fuga de gás na história, e as actividades curatoriais espontâneas que emergiram nos media sociais a esse respeito: um artigo na Wikipédia, uma galeria de fotos no Flickr, vídeos no Youtube, bem como a existência de blogs e tweets e a criação de um grupo no Facebook, que não só mantém a memória do acidente viva, como esta continua a mudar, através da actualização constante da informação, mantendo a narrativa da contaminação do Bhopal e o seu legado relevante hoje enquanto "lição" que pode ser aprendida no presente e para as futuras gerações.

As colecções de arte, ciência, natureza ou história convivem hoje com inúmeros dispositivos tecnológicos e 
interactivos que proporcionam novas experiências interpretativas dos locais patrimoniais, e que permitem aceder a variada e complexa informação digital sobre estes nos seus espaços ou remotamente, e participar da sua construção. A convergência de conteúdos ricos com sistemas de divulgação móveis e multimodais, aproximam-nos da visão de museu ubíquo, presente em todos os tempos e lugares, uma realidade cada vez mais presente, em que os museus como plataformas comunicacionais (Castells, 2001), são territórios propícios para o encontro e para a convivência e trocas culturais e sociais, zona de contacto entre diferentes culturas, local de múltiplas vozes e interpretações, e onde se questiona a memória, a história e a contemporaneidade.

\section{Bibliografia}

Andersen, P. (2007). What is Web 2.0? Ideas, technologies and implications for education. (Vol. 1, No. 1). Bristol, UK: JISC. Acedido em Junho 2014, em: http://www.jisc.org.uk/media/documents/techwatch/tsw0701 b.pdf

Bannier, S., \& Vleugels, C. (2010, April). how Web 3.0 Combines user-generated and Machine-generated Content. In Papers presented at the Transforming Culture in the Digital Age International Conference in Tartu, 14-16 April 2010 (p. 69). Acedido em Fevereiro de 2015, em: http://dspace.ut.ee/bitstream/handle/10062/14768/transform _book.pdf

Barry A., Creating A Virtuous Circle Between A Museum's On-line And Physical Spaces, in J. Trant and D. Bearman (eds.). Museums and the Web 2006: Proceedings, Toronto: Archives \& Museum 
Cadernos de Sociomuseologia- $\mathrm{n}^{\circ} 5$-2015

Informatics, published March 1, 2006 at http://www.archimuse.com/mw2006/papers/barry/barry.html Bradburne, J. (2008). Forward, In.: Tallon, L. \& Walker, K. Digital Technologies and the Museum

Experience. Handheld Guides and other Media. U.K. : Altamira Press

Bruns, A. (2007) Produsage: Towards a Broader Framework for User-Led Content Creation. In Proceedings Creativity \& Cognition 6, Washington, DC. Acedido em Fevereiro de 2015, em: http://eprints.qut.edu.au/6623/1/6623.pdf

Castells, M. (2001). Museus na Era da Informação. Conectores culturais de tempo e espaço. ICOM News Special Issue 2001.

Foucault, M. (1998). Different Spaces. In: Foucault, M. Essential Works of Foucault 1954-1984, Vol. 2, New York: The New York Press. pp.175-185. Acedido em Março 2014, em: http://www.english.upenn.edu/ cavitch/pdflibrary/Foucault_Different.pdf

Giaccardi, E. (2012) Reframing heritage in a participatory culture. In.: Giaccardi, E. (Ed.). Heritage and social media: Understanding heritage in a participatory culture. London: Routledge.

Hazan, S. (2010). A Crisis of Autorithy. In.: Cameron, F. \& Kenderdine,. (ed.) Theorizing Digital cultural Heritage. A Critical Discourse. Cambridge, Massachusetts / London, England: The MIT Press

Huyssen, A. (2014). Políticas de Memória no Nosso Tempo. Lisboa: Universidade Católica Editora Jones, K. (2008). The Transformation of the digital Museum. In.: Marty, P. F., \& Jones, K. B. (ed.). Museum informatics: people, 
information, and technology in museums. New York \& London: Taylor \& Francis.

Lipovetsky, G. \& Serroy, J. (2008). A Cultura-Mundo Resposta a uma Sociedade Desorientada. Lisboa: Edições 70

Liu, S. (2012). Socially Distributed Curation of the Bhopal Disaster. A case of grassroots heritage in the crisis contex. In.: Giaccardi, E. (Ed.). Heritage and social media: Understanding heritage in a participatory culture. Routledge.

Macdonald, G. F. \& Alsford, S. (1997). Towards The Meta Museum. In.: Jones-Garmil, K. (ed.) The Wired Museum: Emerging Technology and Changing paradigms. Washington DC: American Association of Museums. Citado por Black, G. (2012). Transforming Museums In The Twenty-first Century. London and New York: Routledge Taylor \& Francis Group

Marty, P. (2008). An Introduction to Museum Informatics. In.: Marty, P. F., \& Jones, K. B. Museum informatics: people, information, and technology in museums (Vol. 2). New York \& London: Taylor \& Francis.

O'Reilly, T. (2005) What Is Web 2.0,? O'Reilly Network. Acedido em Dezembro de 2014, em: http://www.oreillynet.com/pub/a/oreilly/tim/news/2005/09/ 30/what-is-web-20.html/

Östman, S. (2010, April). Life Publishing on the internet-a playful field of life-telling. In Papers presented at the Transforming Culture in the Digital Age International Conference in Tartu, 14-16 April 2010 (p. 161). Acedido em Fevereiro de 2015, em: http://dspace.ut.ee/bitstream/handle/10062/14768/transform _book.pdf

Toffler, A. (1980). The Third Wave. New york: Bantam Books

Parry, R. (2008). Afterword: The Future in Our Hands? Putting Potencial into Practise. In: Tallon, L. \& Walker, K. (2008). Digital 
Cadernos de Sociomuseologia- n 5 -2015

Technologies and the Museum Experience. Handheld Guides and other Media. Altamira Press: U.K.

Paul, C. (2005). Challenges for a Ubiquitous Museum: Presenting and Preserving New Media. Acedido em Março de 2015, em:

http://www.mediaarthistory.org/refresh/Programmatic\%20key \%20texts/pdfs/Paul.pdf

Peacock, D. (2008). The Information Revolution in the museum. In.: Marty, P. F., \& Jones, K. B. (ed.). Museum informatics: people, information, and technology in museums . New York \& London: Taylor \& Francis.

Pearce, S. M. (1994). Thinking about things. Interpreting objects and collections, 125-132. Acedido em Abril 2015, em: http://lexus.ischool.utexas.edu/Winget_Megan/2010/Fall/INF3 81/Readings/Pearce_ThinkingAboutThings.pdf

Rheingold, H. (2002). Smart mobs: The next Social Revolution. Cambridge, Mass: Basic Books

Ross, M. (2004). Interpreting the new museology. Museum and society, 2(2), 84-103. Acedido em Fevereiro de 2014, em: https://www2.le.ac.uk/departments/museumstudies/museums ociety/documents/volumes/ross.pdf

Russo, A.; Watkins, J.; Kelly, L. \& Chan, S. (2006) How will social media affect museum communication? In.: Proceedings Nordic Digital Excellence in Museums (NODEM), Oslo, Norway.

Acedido em Janeiro de 2015, em: http://eprints.qut.edu.au/6067/1/6067_1.pdf

Russo, A. (2012). The Rise of the "media museum" Creating interactive cultural experiences trough social media. In.: Giaccardi, E. (Ed.). Heritage and social media: Understanding heritage in a participatory culture. Routledge.

Shahani, L., Economou, M., \& Nikonakou, N. (2008). Museums curating online content using Web 2.0: Making cultural 


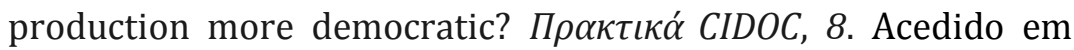
Dezembro de 2014, em:

https://www.academia.edu/397237/MUSEUMS_CURATING_ON LINE_CONTENT_USING_WEB_2.0_MAKING_CULTURAL_PRODUC TION_MORE_DEMOCRATIC

Shirky, C. (2008). Here Comes Everybody: The Power Of Organizing Without Organizations. New York: Penguin Press. Acedido em Abril de 2015, em : https://uniteyouthdublin.files.wordpress.com/2015/01/here_co mes_everybody_power_of_organizing_without_organizations.pdf Simon, Nina. (2010). The Participatory Museum. California: Museum 2.0

Sumption, K. (2006). In Search Of The Ubiquitous Museum: Reflections Of Ten Years Of Museums And The Web. Acedido em Fevereiro de 2015, em: http://www.archimuse.com/mw2006/papers/sumption/sumpti on.html

Weil, S. E. (1999). From being about something to being for somebody: The ongoing transformation of the American museum. Daedalus, 229-258. Acedido em Janeiro de 2014, em: http://isites.harvard.edu/fs/docs/icb.topic862568.files/Supple mentary\%20Readings/Weil\%201999.pdf Zorich, D. (2008). Information Policy in Museums. In.: Marty, P. F., \& Jones, K. B. (ed.). Museum informatics: people, information, and technology in museums . New York \& London: Taylor \& Francis. 